\title{
Is the World Prepared for the New Immigration Wave? \\ Edmond Puca*
}

Department of Infectious Diseases, University Hospital Center "Mother Teresa", Tirana, Albania

"Corresponding author: Edmond Puca, Department of Infectious Diseases, University Hospital Center "Mother Teresa", Tirana, Albania, Tel: 0672058624; E-mail: edmond_puca@yahoo.com

Received date: December 30, 2015; Accepted date: December 31, 2015; Published date: December 31, 2015

Copyright: (c) 2015 Puca E. This is an open-access article distributed under the terms of the Creative Commons Attribution License, which permits unrestricted use, distribution, and reproduction in any medium, provided the original author and source are credited.

\section{Editorial}

Immigration has become a major and even extremely multifaceted problem nowadays. It accounts of political, economic, social and ethnic problems, but in this article we want to draw the attention on the medical problems that it can present. Every human being has the right to choose how to live his/her life, but through this choice other problems can be raised. On this perspective poorly controlled movements or even worse, the uncontrolled ones, modify the prevalence of morbidity in general and in particular the prevalence of infectious diseases. Developed countries have been and continue to be under continuous pressure of immigrants from poor and unsafe country. About 230 million migrants have been registered at the end of 2013 [1].

Immigrants generally originate from regions where infectious diseases continue to pose a significant challenge for the national infectious diseases control. While developed countries have standardized the relations patient/infectious diseases/reporting cases. In this regard, we can say that the latter have eliminated or have a few cases of the diseases that continue to predominate in the developing countries or in developed countries. Tuberculosis can be an example of the situation described above. After 1993, tuberculosis has been classified as a global emergency. It is also known that in the least developed countries it constitutes a major public health problem. Tuberculosis, if not diagnosed and treated, is associated with longer term risk for disease. As a consequence from these regions people and infectious diseases will emigrate together. The actual case is the immigrant crisis which occurred during 2015. In Europe a wave of immigrants, mainly from Africa and Asia erupted and in certain moments it became unmanageable. Since the origin of these immigrants is also from afro-asiatic countries the diseases that they carry are quite diverse. Currently the largest number of refugees in Europe constitutes of Syrians, but also immigrants from Africa and the least developed countries in the Balkans. It is estimated that some 6.5 million people are displaced within the country. More than 4.2 million Syrians have crossed borders; Turkey hosts more than 2.1 million refugees, Lebanon 1 million, Jordan 630,000 and Iraq 235,000 and there are approximately 682,000 asylum applications in Europe [2] .

Syrians among others are faced with a long war which has created conditions for a reduced hygiene, people living in camps within or in the neighboring countries, lacking care or prevention for infectious diseases. The lack of shelter, insufficient access to clean water, food, sanitation, and health care associated with mass migration can quickly degrade the health of a population. Measles, hepatitis, leishmaniasis, poliomyelitis, meningitis, and scabies have spread through vulnerable populations in Syria and refugee camps in neighboring countries, health creating a crisis that will require immense resources to address [3]. In these conditions it is difficult to say if an adequate vaccination for infectious diseases has been done. Thus raising the question: are we prepared for such a global crisis? Is our health system prepared with personnel, equipment or vaccine for such a large and immediate immigrants flow? During almost more than half a century, the vaccination programs in developed countries have shown to be effective. However, these successes have not been uniform and infectious diseases continue to remain the leading causes of mortality and morbidity in some regions of the world. Children with unclear immunization histories will need vaccination, while adults may already have immunity to measles, mumps, rubella, and other diseases. Current examples to illustrate this include malaria, which is historically linked with the return of visitors and tourists from areas of endemicity to non-endemic areas [4]. On the other hand there are our diagnostic and therapeutic management possibilities of immigrant patients. Doctors from developed countries are often unfamiliar with the infectious diseases that these patients present [5]. Thus to them the establishment of the diagnosis of infectious diseases will be a real challenge. Intestinal parasites such as strongyloidiasis and schistosoma are common infections in refugee populations from sub-Saharan Africa and South Asia and can lead to anemia as well as liver and urological complications. But immigration is not just a matter of several months or years. Immigrants present an issue to be resolved in time, because a part of them will manage to stabilize in the host countries, others could seek family reunion and later they will be visited by their relatives. So the situations presented are not so simple to be solved.

Thus seeing this diversity of immigrants movement expected in the subsequent decades, the map of infectious diseases especially in Europe, but also in the whole world will have a new look.

\section{References}

1. United Nations, Department of Economic and Social Affairs, Population Division (2013) Trends in International Migrant Stock: The 2013 Revision.

2. Leblebicioglu H, Ozaras R (2015) Syrian refugees and infectious disease challenges. Travel Medicine \& Infectious Disease 13: 443-444.

3. Sharara SL, Kanj SS (2014) War and infectious diseases: Challenges of the syrian civil war. PLoS Pathog 10: e1004438.

4. Gushulak BD, MacPherson DW (2004) MacPherson. Globalization of infectious diseases: the impact of migration. Clin Infect Dis 38: 1742-1748.

5. Maroun E, Kushawaha A, El-Charabaty E, Mobarakai N, El-Sayegh S (2011) Fulminant Leptospirosis (Weil's disease) in an urban setting as an overlooked cause of multiorgan failure: a case report. Journal of Medical Case Reports 5: 7. 